\title{
FlexibleSUSY: Precise automated calculations in any BSM theory
}

\author{
Peter Athron* \\ ARC Centre of Excellence for Particle Physics at the Terascale, School of Physics, Monash \\ University, Melbourne, Victoria 3800, Australia \\ E-mail: peter.athron@coepp.org.au
}

Markus Bach

Dylan Harries

Wojciech Kotlarski

Thomas Kwasnitza

Jae-hyeon Park

Tom Steudtner

Dominik Stöckinger

Alexander Voigt

Jobst Ziebell

\begin{abstract}
FlexibleSUSY is a software package for various calculations in any model of physics beyond the standard model (not just any supersymmetric model). FlexibleSUSY can solve boundary value problems and uses this to find $\overline{D R} / \overline{M S}$ parameters and calculate the Higgs and BSM particle masses, as well as other observables. FlexibleSUSY is designed to be adaptable, fast, precise and reliable. We describe FlexibleSUSY with particular emphasis on recent developments and the state of the art Higgs mass calculations it can perform. We also show some applications to illustrate how it can be used to obtain interesting physics results with the highest precision possible and with remarkable speed.
\end{abstract}

The 39th International Conference on High Energy Physics (ICHEP2018)

4-11 July 2018

Seoul, South Korea

${ }^{*}$ Speaker. 


\section{FlexiblesUSY}

A major difficulty in investigating the phenomenology of a novel extension of the standard model is that for meaningful predictions of observables one must perform many dedicated calculations, often at the 1- or 2-loop level and implement these calculations in a programming language so that the parameter space can be explored. Therefore automation of these steps can be extremely useful for phenomenology. FlexibleSUSY $[1,2,3]$ is an easy-to-use software package which generates a $\mathrm{C}++$ spectrum generator and $\mathrm{C}++$ calculations of observables, for any BSM model. Here we will describe FlexibleSUSY and many of its uses. FlexibleSUSY uses SARAH [4] to obtain a number of model-specific expressions and corrections. Analytic expressions obtained in Mathematica are then translated to $\mathrm{C}++$ and implemented in a code for spectrum generator ${ }^{1}$ and observable calculations which is fast, adaptable and reliable. For any of the many distributed model files, the user can immediately create a spectrum generator with three simple commands, e.g.

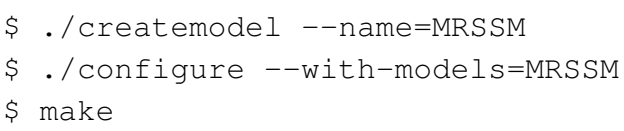

The FlexibleSUSY spectrum generator may then be run with the following:

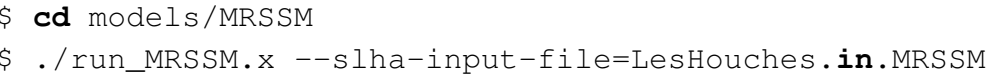

In the MSSM and NMSSM FlexibleSUSY can output SLHA [8] and SLHA2 [9] files for interfacing with other codes. In other models a similar SLHA-like interface can be used if the other codes have also been setup to work with that model by using SARAH, with the same SARAH model files as FlexibleSUSY. For example FlexibleSUSY linked to micrOMEGAs [10] can be used to investigate dark matter [11], with the parameter space sampled efficiently by also interfacing to MultiNest [13]. FlexibleSUSY can also be used in the global fitting tool GAMBIT [14] where it is interfaced with a huge array of observable calculators and samplers.

2-loop RGEs, and full 1-loop self-energy and tadpole corrections are obtained using modelspecific analytical expressions that are generated using SARAH. In addition FlexibleSUSY also implements its own corrections that are also applied to all models. These include a 1-loop calculation of threshold corrections for extracting $\alpha_{s}\left(m_{Z}\right)$ and $\alpha_{e}\left(m_{Z}\right)$ in the considered extension of the SM, a new (partial 2-loop) calculation of the weak mixing angle $\sin \theta_{W}$ which allows us to obtain the $S U(2)_{W}$ and $U(1)_{Y}$ gauge couplings, $g$ and $g^{\prime}$ respectively, and pure QCD 2-loop corrections to the running top and bottom Yukawa couplings. With these precision corrections FlexibleSUSY provides state-of-the-art predictions of the mass spectra in any novel extension of the SM chosen by the user.

However in addition to the calculations applicable to any model, FlexibleSUSY contains many model specific corrections where there have been recent improvements. Therefore FlexibleSUSY includes: 3-loop RGEs in the SM and MSSM; 2-loop (SUSY) QCD corrections to top, bottom

\footnotetext{
${ }^{1}$ This spectrum generator contains some numerical routines from SOFTSUSY [6].
} 
Yukawas in SM and MSSM [16]; 2-loop (SUSY) QCD corrections to strong gauge coupling in SM, MSSM [16]; 2-loop fixed order Higgs mass corrections SM, MSSM, NMSSM; 3-loop fixed order Higgs mass corrections SM, MSSM (via Himalaya [17]); and 3-loop Higgs mass corrections in Split-MSSM [18]. Thus FlexibleSUSY also provides state-of-the-art calculation in well-studied models like the MSSM, NMSSM.

Since the Higgs boson mass has been measured with an uncertainty is that is already significantly smaller than the theoretical uncertainty, FlexibleSUSY has a number of very sophisticated Higgs mass calculations. For all models FlexibleSUSY implements the FlexibleEFTHiggs [2] algorithm, which resums logarithms at NLL precision ensuring precise predictions when the new physics scale is high, while matching the 1-loop precision ${ }^{2}$ of the fixed-order calculations when the new physics appears at the electroweak scale ${ }^{3}$. We therefore recommend users employ this approach in all cases unless there is a more precise model-specific EFT or fixed-order calculation (depending on the new physics scale) available. For specific models we have additional fixed order corrections mentioned above, allowing an improved prediction of the Higgs mass, for example at the 3-loop level in the MSSM via Himalaya [17]. We also provide special model files where matching conditions between the specific high scale and low scale model are implemented. The FlexibleSUSY spectrum generators created from these specific model files then perform precise Higgs mass calculations with resummed logs. Currently FlexibleSUSY has:

- HSSUSY [20]: MSSM calculation assuming a SM EFT, predicts $M_{h}$ at full NLO with full NLL ressummation + NNLO with NNLL ressumation at $\mathscr{O}\left(\alpha_{t}\left(\alpha_{s}+\alpha_{t}\right)+\left(\alpha_{t}+\alpha_{b}\right)^{2}+\right.$ $\left.\alpha_{\tau} \alpha_{b}+\alpha_{\tau}^{2}\right)+$ (very recent) $\mathrm{N}^{3} \mathrm{LO}$ corrections with $\mathrm{N}^{3} \mathrm{LL}$ at $\mathscr{O}\left(\alpha_{t} \alpha_{s}^{2}\right)$ resummation ${ }^{4}$.

- SplitMSSM: MSSM calculation assuming a SplitSUSY EFT, predicts $M_{h}$ at full NLO + NNLO $\mathscr{O}\left(\alpha_{t} \alpha_{s}\right)$ with full NLL resummation.

- THDMIIMSSMBCFull: MSSM calculation assuming a THDMII EFT, predicts $M_{h}$ at full NLO (+ partial NNLO $\left.\mathscr{O}\left(\alpha_{t} \alpha_{s}\right)\right)$ with full NLL resummation.

- HTHDMI IMSSMBC: MSSM calculation assuming an EFT with THDMII + light Higgsinos states, predicts $M_{h}$ at full NLO (+ partial NNLO $\mathscr{O}\left(\alpha_{t} \alpha_{s}\right)$ ) with full NLL resummation.

- HGTHDMI IMSSMBCF U I: MSSM calculation assuming an EFT with THDMII + light Higgsino + light gaugino states, predicts $M_{h}$ at full NLO (+ partial NNLO $\left.\mathscr{O}\left(\alpha_{t} \alpha_{s}\right)\right)$ with full NLL resummation.

As well as the spectrum and Higgs mass calculations, FlexibleSUSY has also implemented additional observable calculations. FlexibleSUSY includes a FlexibleAMU calculation of the anomalous magnetic moment of the muon at 1-loop, including fermions and scalars in the loops. Additional 2-loop contributions and vector contributions will be included in a future update. FlexibleSUSY also includes a 1-loop calculation of lepton EDMs which can provide important constraints when the parameters include complex phases. The new extraction of $\sin \theta_{W}$ also

\footnotetext{
${ }^{2}$ A 2-loop fixed order calculation for any model, using a SARAH extension [19] is currently under development.

${ }^{3} \mathrm{~A}$ version of this procedure with 2-loop matching to provide NNLL resummation is currently under development.

${ }^{4}$ With these recent corrections [21] this C++ Higgs mass calculator is the most precise EFT calculation of the Higgs mass available for the MSSM, with a higher precision than the next-best competitor SusyHD [22].
} 
allows us to predict the mass of the $W$ boson, instead of using it as an input to obtain $\sin \theta_{W}$. FlexibleSUSY can also provide the effective vertices for $h \rightarrow \gamma \gamma$ and $h \rightarrow g g$ decays (where $h$ can be the SM Higgs or any neutral scalar in the SM extension). A full loop-level calculation of Higgs decays and decays of BSM states is under development, with a release anticipated soon.

\section{Conclusions}

FlexibleSUSY is a software package for calculating mass spectra and a growing set of observables in a fast, flexible, precise and robust manner. FlexibleSUSY is easy to use and can be readily interfaced with other codes to do detailed phenomenological investigations, and even global fits in both novel and well-known extensions of the standard model.

\section{References}

[1] P. Athron et al. Comput. Phys. Commun. 190, 139 (2015) doi:10.1016/j.cpc.2014.12.020

[2] P. Athron et al. JHEP 1701, 079 (2017) doi:10.1007/JHEP01(2017)079

[3] P. Athron et al. Comput. Phys. Commun. 230, 145 (2018) doi:10.1016/j.cpc.2018.04.016

[4] F. Staub, Comput. Phys. Commun. 181, 1077 (2010) doi:10.1016/j.cpc.2010.01.011

[5] F. Staub, Comput. Phys. Commun. 182, 808 (2011) doi:10.1016/j.cpc.2010.11.030

[6] B. C. Allanach, Comput. Phys. Commun. 143, 305 (2002) doi:10.1016/S0010-4655(01)00460-X [hep-ph/0104145].

[7] B. C. Allanach et al. Comput. Phys. Commun. 185, 2322 (2014) doi:10.1016/j.cpc.2014.04.015

[8] P. Z. Skands et al., JHEP 0407, 036 (2004) doi:10.1088/1126-6708/2004/07/036 [hep-ph/0311123].

[9] B. C. Allanach et al., Comput. Phys. Commun. 180, 8 (2009) doi:10.1016/j.cpc.2008.08.004

[10] G. Belanger et al., Comput. Phys. Commun. 149, 103 (2002) doi:10.1016/S0010-4655(02)00596-9 [hep-ph/0112278].

[11] P. Athron et al. Phys. Lett. B 760, 19 (2016) doi:10.1016/j.physletb.2016.06.040

[12] P. Athron et al. JHEP 1612, 128 (2016) doi:10.1007/JHEP12(2016)128

[13] F. Feroz et al. Mon. Not. Roy. Astron. Soc. 398, 1601 (2009) doi:10.1111/j.1365-2966.2009.14548.x

[14] P. Athron et al. [GAMBIT Collaboration], Eur. Phys. J. C 77, no. 11, 784 (2017) Addendum: [Eur. Phys. J. C 78, no. 2, 98 (2018)] doi:10.1140/epjc/s10052-017-5513-2, 10.1140/epjc/s10052-017-5321-8

[15] P. Athron et al., Eur. Phys. J. C 78, no. 1, 22 (2018) doi:10.1140/epjc/s10052-017-5390-8

[16] B. C. Allanach et al. Comput. Phys. Commun. 189, 192 (2015) doi:10.1016/j.cpc.2014.12.006

[17] R. V. Harlander et al. Eur. Phys. J. C 77, no. 12, 814 (2017) doi:10.1140/epjc/s10052-017-5368-6

[18] K. Benakli et al. JHEP 1405, 113 (2014) doi:10.1007/JHEP05(2014)113

[19] M. Goodsell et al. Eur. Phys. J. C 75, no. 6, 290 (2015) doi:10.1140/epjc/s10052-015-3494-6

[20] E. Bagnaschi et al. JHEP 1603, 158 (2016) doi:10.1007/JHEP03(2016)158

[21] R. V. Harlander et al. Eur. Phys. J. C 78, no. 10, 874 (2018) doi:10.1140/epjc/s10052-018-6351-6

[22] J. Pardo Vega and G. Villadoro, JHEP 1507, 159 (2015) doi:10.1007/JHEP07(2015)159 